\title{
Diabetische Retinopathie bei Patienten mit Diabetes mellitus
}

\author{
Olga Simó-Servat ${ }^{a, b} \quad$ Cristina Hernández ${ }^{a, b}$ Rafael Simóa, b \\ aDiabetes and Metabolism Research Unit, Vall d'Hebron Research Institute, Barcelona, Spanien; \\ ${ }^{b}$ Centro de Investigación Biomédica en Red de Diabetes y Enfermedades Metabólicas Asociadas (CIBERDEM), \\ Instituto de Salud Carlos III (ICSIII), Madrid, Spanien
}

\section{Schlüsselwörter}

Diabetische Retinopathie · Erblichkeit · glykämische Variabilität · mikro- und makrovaskuläre Komplikationen · kognitive

Einschränkungen · Neurodegeneration

\section{Zusammenfassung}

Die diabetische Retinopathie (DR) ist die häufigste Komplikation von Diabetes mellitus. Hauptrisikofaktoren sind die Krankheitsdauer, schlechte Blutzuckereinstellung und das Vorliegen einer Hypertonie. Allerdings fällt das Risiko sehr unterschiedlich aus, was dafür spricht, dass bei der Entwicklung einer DR andere Faktoren, wie beispielsweise die Erblichkeit oder die glykämische Variabilität eine wichtige Rolle spielen. Einem anderen wichtigen Konzept zufolge handelt es sich bei der DR um einen unabhängigen Prädiktor mikrovaskulärer und makrovaskulärer Komplikationen. Daher muss bei der Bewertung des kardiovaskulären Risikos eines diabetischen Patienten das Vorliegen einer DR berücksichtigt werden. Darüber hi- naus kann die Beurteilung der retinalen Neurodegeneration helfen, Diabetiker zu identifizieren, bei denen das Risiko einer kognitiven Einschränkung besteht, einer Komplikation, die bei der Population der Typ-2-Diabetiker zunehmend auftritt. Das Bewusstsein für eine etwaige DR hat bei der Beurteilung diabetischer Patienten auch therapeutische Implikationen. So kann beispielsweise nach einer raschen Verbesserung der Blutzuckerwerte eine Verschlechterung der DR auftreten. Insgesamt enthält die vorliegende Arbeit eine kritische Übersicht über die Bedeutung der DR in der allgemeinen Versorgung von Patienten mit Diabetes.

(c) 2019 S.Karger GmbH, Freiburg

\section{Das Ausmaß des Problems}

Die diabetische Retinopathie (DR) ist die häufigste Komplikation von Diabetes mellitus und stellt in den Industrieländern weiterhin die Hauptursache von vermeidbarer Erblindung der Bevölkerung im arbeitsfähigen Alter dar [1-3]. Die DR wurde lange Zeit als mikrovaskuläre Komplikation von Diabetes angesehen; es liegen jedoch zunehmend Hinweise vor, nach denen es bereits frühzeitig während der Pathogenese zu einer Neurodegeneration kommt $[4,5]$. Tatsächlich lassen sich bei Patienten ohne Anhalt für mikrovaskuläre Veränderungen Anomalien der Netzhautfunktion feststellen, und die American Diabetes Association (ADA) hat die DR kürzlich als hoch spezifische neurovaskuläre Komplikation eingestuft [6].

Die DR und ihr Fortschreiten sind mit einem erheblichen Anstieg der Gesundheitskosten verbunden [7]. Da die DR die häufigste Komplikation von Diabetes ist und den Annahmen zufolge von 415 Millionen Fällen im Jahr 2015 auf 642 Millionen im Jahr 2040 zunehmen wird, wird die DR im Zukunft ein noch größeres Problem darstellen [8].

\section{KARGER}

Fax +497614520714

information@karger.com

www.karger.com

\section{(c) 2019 S. Karger GmbH, Freiburg}

Accessible online at:

www.karger.com/kop
Rafael Simó, MD, PhD

Diabetes and Metabolism Research Unit

Vall D'Hebron Research Institute (VHIR)

Pg. Vall d'Hebron 119-129, 08035 Barcelona, Spanien

rafael.simo@vhir.org 
Die derzeitigen Therapien zielen auf die Spätstadien der DR ab, in denen die Sehkraft bereits erheblich beeinträchtigt ist. Ein besseres Verständnis der Pathogenese der DR würde die Entwicklung neuartiger und wirksamerer präventiver/interventioneller Strategien für die frühen Stadien der DR ermöglichen $[9,10]$. Der vorliegende Artikel liefert eine kritische Übersicht über den aktuellen Wissensstand zum bidirektionalen Einfluss der DR auf den Diabetes und die damit verbundenen Komplikationen.

\section{Risikofaktoren für die Entwicklung einer DR}

Die Hauptrisikofaktoren für die Entwicklung einer DR sind die Krankheitsdauer, eine schlechte Blutzuckereinstellung (hohe HbAlc-Werte) und das Vorliegen einer Hypertonie. $\mathrm{Zu}$ den weiteren Risikofaktoren gehören ein hoher Body-Mass-Index (BMI), Pubertät,Schwangerschaft sowie eine Kataraktoperation [3].

Es liegen solide Hinweise vor, die den Zusammenhang zwischen den Blutzuckerwerten und der Entwicklung und Progression einer DR belegen. In der UKPDS (UK Prospective Diabetes Study)Population von Patienten mit Typ-2-Diabetes war die intensivierte Therapie im Vergleich zum konventionellen Blutzuckermanagement mit einer 39\%igen Risikoreduktion für eine Laser-Photokoagulation verbunden [11]. In der DCCT (Diabetes Control and Complications Trial)-Population von Patienten mit Typ-1-Diabetes reduzierte die straffe Blutzuckerkontrolle gegenüber der weniger straffen Blutzuckerkontrolle das Risiko für eine neu auftretende Retinopathie um 76\% und für die Progression einer bestehenden Retinopathie um 54\% [12]. Kürzlich veröffentlichten Berichten zufolge scheint sich die proliferative DR bei Typ1-Diabetikern für bis zu 20 Jahre verhindern zu lassen, wenn als Therapieziel HbAlc-Werte von weniger als 7,6\% (60 mmol/mol) angestrebt werden [13]. Die Serum-Lipidwerte scheinen weniger Einfluss auf die Entwicklung einer proliferativen DR oder eines diabetischen Makulaödems (DMÖ) zu haben [14, 15]. Es liegen jedoch zunehmend Belege dafür vor, dass die nicht-herkömmlichen Lipidmaße (beispielsweise Apolipoproteine A und B) stärkere Risikomarker für eine DR sind als die Gesamtcholesterinund Triglyzeridwerte [16, 17].

Der Zusammenhang zwischen Hypertonie und DR ist ebenfalls bekannt. Die UKPDS zeigte, dass die Gruppe, die einer straffen Blutdruckkontrolle zugewiesen worden war, nach 9-jährigem Followup eine 34\%ige Risikoreduktion im Anteil der Patienten, die eine Verschlechterung der Retinopathie um 2 Stufen zeigten ( $p=$ $0,0004)$, aufwies, und ein um $47 \%$ geringeres Risiko ( $p=0,004)$ für eine Verschlechterung der Sehschärfe um 3 Linien der ETDRSTafel [11]. Eine kürzlich durchgeführte populationsbasierte Querschnittsstudie (13 473 Patienten) ergab, dass Hypertonie ein unabhängiger Risikofaktor sowohl für eine leichte bis mäßige DR als auch für die potenziell zum Verlust des Sehvermögens führende DR ist [18]. Die Leitlinien des Joint National Committee 8 empfehlen eine Blutdruckeinstellung auf Werte von $<140 / 90 \mathrm{mmHg}$ [19]. Nach den aussagekräftigsten aktuellen Belegen der ophthalmologischen Literatur ist eine Änderung dieser Empfehlung für Patienten mit bestätigter DR nicht mit einem Vorteil verbunden.
Zirkulierende Zytokine, die bei Typ-2-Diabetikern erhöht sind, können außerdem zu einer vermehrten Gefäßleckage führen, doch ist ihre Beteiligung an der Entwicklung einer DR und eines DMÖ noch unklar. In diesem Zusammenhang ist zu beachten, dass die lokale Synthese durch die Retina, hauptsächlich NetzhautpigmentepithelundGliazellen, dieHauptquelleproinflammatorischer Zytokine ist. Daher sind intraokular applizierte entzündungshemmende Wirkstoffe, wie beispielsweise Kortikosteroide, wirksamer in der Behandlung eines DMÖ als systemische Wirkstoffe $[9,20]$.

Zwar sind die oben beschriebenen Risikofaktoren an der Entwicklung und Progression der DR beteiligt, doch zeigen klinische Studien mit Diabetikern, dass erhebliche Unterschiede im Hinblick auf das Auftreten und den Schweregrad der DR bestehen. Diese sind durch die bekannten Risikofaktoren nicht vollständig zu erklären, und alle Kliniker sind sich bewusst, dass es eine Untergruppe von Patienten mit schlechter Blutzuckereinstellung und/oder unkontrolliertem Blutdruck gibt, die keine DR entwickelt. Demgegenüber gibt es Patienten mit sehr guter Blutzuckereinstellung, die keine Hypertonie haben, bei denen eine DR auftritt. Tatsächlich zeigte die DCCT/EDIC-Forschungsgruppe, dass die HbA1c-Werte 11\% des DR-Risikos erklärten und dass die ungeklärten 89\% der Risikoschwankung durch Faktoren des diabetischen Milieus bedingt sind, die vom mittleren HbAlc-Wert nicht erfasst werden [21].

Diese Daten sprechen dafür, dass andere Faktoren für die Neigung, diese Diabetes-Spätkomplikation zu entwickeln, eine wichtige Rolle spielen. In diesem Zusammenhang wurden Schätzwerte zur Erblichkeit der proliferativen DR zwischen 25\% und 50\% berichtet $[22,23]$. Die Studie mit eineiigen Zwillingen stellte eine Konkordanz für die DR fest (68\% bei Typ-1- und 95\% bei Typ-2-Diabetes) [24]. In der DCCT-Kohorte betrug die Odds Ratio für die Entwicklung einer schweren Retinopathie 3,1, wenn ein Angehöriger ebenfalls eine Retinopathie hatte [25]. Andere Studien zum familiären Risiko kamen zu ähnlichen Ergebnissen [26-28].

Die Schwankungen der Plasmaglukosewerte sind ein weiterer Grund dafür, dass der HbAlc-Wert ein schwacher Prädiktor für die Entwicklung oder Progression einer DR ist. Mit der Einführung der kontinuierlichen Glukosemessung (continuous glucose monitoring, CGM), die das Blutzuckerprofil über mehrere Tage erfasst, war es möglich, Parameter der Blutzuckermessung zu entwickeln, die wertvolle Informationen liefern, welche über die Informationen, die der HbAlc-Wert liefert, hinausgehen. Ein Parameter der CGM ist die «Zeit im Zielbereich» (time in range, TIR). Diese gibt an, über wie viel Zeit die gemessenen Blutzuckerwerte innerhalb des angestrebten Bereichs (üblicherweise 3,9-10,0 $\mathrm{mmol} / \mathrm{l}$ ) lagen und liefert wertvolle Informationen darüber, ob sich die Häufigkeit und Dauer von hypo- oder hyperglykämischen Episoden mit der Zeit verbessern. In einer kürzlich veröffentlichten Studie war die TIR nach Korrektur hinsichtlich Alter, Geschlecht, BMI, Dauer des Diabetes, Blutdruck, Lipidprofil und HbA1c-Wert mit allen Stadien der DR assoziiert [29]. Ferner haben eine systematische Übersichtsarbeit und eine Metaanalyse 
gezeigt, dass die Schwankungen der Nüchtern-Blutzuckerwerte eng mit dem Retinopathierisiko assoziiert waren [30].

Und schließlich könnten epigenetische Veränderungen ein Schlüsselmechanismus für das Phänomen des so genannten «metabolischen Gedächtnisses» sein, das die Langzeiteffekte des metabolischen Zustands, der über einen mehrere Jahre in der Vergangenheit liegenden Zeitraum bestand, erklären könnte [31].

\section{DR - Die Büchse der Pandora diabetischer Komplikationen}

Das Vorliegen einer DR zeigt an, dass die Mikrozirkulation bereits durch das diabetische Milieu gestört ist, und kann daher als zuverlässiger Biomarker für die schädlichen Auswirkungen des Diabetes bei einem bestimmten Patienten angesehen werden. Tatsächlich handelt es sich um einen der wichtigsten Indikatoren für die Planung einer personalisierten Behandlung. Eine kürzlich durchgeführte systematische Analyse ergab klare Belege für die Zusammenhänge zwischen einer DR und den Komplikationen von Diabetes, einschließlich mikro- und makrovaskulärer Erkrankungen und Ereignisse [32]. Zwar wurden diese Zusammenhänge in vielen Fällen in Querschnittsstudien und retrospektiven Untersuchungen ermittelt, doch konnten etliche in prospektiven Untersuchungen auf Grundlage multivariater Analysen, die hinsichtlich des Einflusses der herkömmlichen oder anderen bekannten Risikofaktoren korrigiert waren, bestätigt werden.

Eine DR erhöht die Wahrscheinlichkeit für eine Nephropathie [33-35] und stellt einen signifikanten und unabhängigen Prädiktor für die Progression einer Mikro- oder Makroalbuminurie dar [33]. Die DR ist mit einer Abnahme der glomerulären Filtrationsrate korreliert [36, 37]. Daher kann eine engmaschige Überwachung von Patienten mit DR auf das Vorliegen einer Albuminurie und gestörten glomerulären Funktion helfen, die Progression zu einer schweren Nierenerkrankung zu verhindern.

Es wurde gezeigt, dass die DR mit 2 Arten von Neuropathie assoziiert ist: der peripheren diabetischen Neuropathie [38, 39] und der kardialen autonomen Neuropathie (CAN) [40, 41]. Derzeit ist nicht bekannt, ob ein Zusammenhang zwischen den neurodegenerativen Veränderungen der Retina und der peripheren Neuropathie besteht. Eine kürzlich durchgeführte Studie mit 27 Typ1-Diabetikern ergab jedoch eine Korrelation zwischen dem Verlust der inneren Retina in der SD-OCT und einer verringerten autonomen Regulation [42].

Neben den mikrovaskulären Komplikationen ist die DR auch mit der Entwicklung von makrovaskulären Komplikationen bei Diabetes assoziiert, insbesondere zerebrovaskulären Komplikationen (Schlaganfall, Hirninfarkt/-blutung), kardiovaskulären Komplikationen (Atherosklerose, kardiovaskuläre Ereignisse und koronare Herzkrankheit) sowie peripheren Komplikationen (Fußulzera, Amputation der unteren Extremität und periphere arterielle Verschlusskrankheit) [32]. Wichtig ist zudem, dass die DR sowie die Mikroalbuminurie und die periphere Neuropathie sehr viel bessere Prädiktoren für kardiovaskuläre Erkrankungen sind als die klassischen Risikofaktoren wie Cholesterin und Hypertonie [43].
Da sowohl die kardiale autonome Neuropathie als auch die DR in verschiedenen prospektiven Studien mit Diabetikern als Prädiktoren der kardiovaskulären Morbidität und Mortalität ermittelt wurden, könnte es interessant sein zu untersuchen, ob das gemeinsame Auftreten beider Erkrankungen mit einem höheren Risiko kardiovaskulärer Komplikationen verbunden ist als das Vorliegen der jeweiligen Komplikation allein. In diesem Fall könnte eine Screeninguntersuchung von Diabetikern mit DR auf CAN helfen, Hochrisikogruppen zu identifizieren, bei denen eine Überwachung und Früherkennung der klinischen Progression zu einer Herzkreislauferkrankung erfolgen sollte.

\section{Bedeutung der DR für die Konzeption klinischer Studien zur Beurteilung einer Herzkreislauferkrankung}

Nachdem Rosiglitazon 2008 wegen des offensichtlichen Zusammenhangs mit einem erhöhten kardiovaskulären Risiko vom Markt genommen worden war, gab es so etwas wie einen Wendepunkt. Im Rahmen des regulatorischen Prozesses veröffentlichte die US-amerikanische Food and Drug Administration (FDA) eine Richtlinie für Sponsoren darüber, wie nachzuweisen ist, dass eine neue antidiabetische Therapie zur Behandlung von Typ2-Diabetikern nicht mit einer inakzeptablen Erhöhung des kardiovaskulären Risikos verbunden ist [44]. Diese FDA-Empfehlung führte dazu, dass die pharmazeutischen Unternehmen Studien konzipierten, die auf die Untersuchung der kardiovaskulären Sicherheit neuer Antidiabetika ausgerichtet waren.

Seither wurden mehrere multizentrische prospektive randomisierte kontrollierte Studien zur Beurteilung der kardiovaskulären Sicherheit von Arzneimitteln zur Behandlung von Typ-2-Diabetes veröffentlicht. Diese hatten einen signifikanten Einfluss auf die neueste Stellungnahme der ADA und der European Association for the Study of Diabetes (EASD) über die Behandlung von Typ-2-Diabetes [45]. Seitdem nachgewiesen wurde, dass SGLT2-Inhibitoren und GLP-1R-Agonisten bei der Senkung der kardiovaskulären Morbidität und Mortalität im Vergleich zu Placebo überlegen sind, spielen diese Arzneimittel in den Therapieleitlinien eine zentrale Rolle. In keiner dieser Studien wurden Vorliegen und Ausmaß der DR bei der Randomisierung einbezogen. Dies stellt ein schwerwiegendes wissenschaftliches Manko dar, das es bei zukünftigen klinischen Studien zu diesem Thema zu berücksichtigen gilt.

\section{DR als Prädiktor für kognitive Einschränkungen und Demenz}

Die Ergebnisse von Längsschnittstudien zeigen, dass der kognitive Abbau bei Typ-2-Diabetikern bis zu doppelt so schnell verläuft wie die normale Alterung, und Diabetiker haben ein erhöhtes Risiko für eine leichte kognitive Störung (mild cognitive impairment, MCI) [46], die eine kognitive Beeinträchtigung gemäß den Standardtests beinhaltet, jedoch keine Auswirkungen auf die Alltagsaktivitäten hat und die einen Übergangszustand zwischen normaler kognitiver Funktion und Demenz darstellt. Darüber hi- 
naus hatten Typ-2-Diabetiker ein fast doppelt so hohes Risiko, eine Alzheimer-Krankheit zu entwickeln wie nach dem Alter vergleichbare Nicht-Diabetiker [47]. Das erhöhte Risiko blieb auch nach Anpassung hinsichtlich vaskulärer Risikofaktoren bestehen. Die jährliche Konversionsrate von MCI zu Demenz liegt in der Allgemeinbevölkerung zwischen 10\% und 30\%, ist jedoch in der Population von Patienten mit Typ-2-Diabetes noch höher [48]. Wegen der Diabetes-Pandemie und dem gleichzeitig zunehmenden Alter der Bevölkerungen weltweit wird zudem die Zahl der Typ-2-Diabetiker mit kognitiven Einschränkungen oder Demenz den Erwartungen zufolge ansteigen. In diesem Zusammenhang ist damit zu rechnen, dass die schwere kognitive Beeinträchtigung zu einer «neuen» Langzeitkomplikation von Diabetes wird, mit dramatischen Folgen für die betroffenen Patienten und ihre Angehörigen und mit erheblichen Auswirkungen auf die Gesundheitssysteme [49]. Daher sind dringend Strategien zur Identifizierung von Diabetikern mit Demenzrisiko erforderlich. Tatsächlich empfiehlt die ADA eine Individualisierung der DiabetesTherapie, die die kognitiven Fähigkeiten der Patienten berücksichtigt [50]. In der klinischen Praxis existieren jedoch keine bekannten phänotypischen Indikatoren oder zuverlässigen Untersuchungen zur Identifizierung von Typ-2-Diabetikern mit MCI.

Die Diagnose einer MCI basiert auf komplexen neuropsychologischen Tests, die für eine Integration in die derzeitigen Standardbehandlungen für Typ-2-Diabetes ungeeignet sind. Kürzlich wurde berichtet, dass die Netzhautsensitivität [51] und die Blickfixierung [52] gemäß Mikroperimetrie hilfreiche Biomarker zur Identifizierung von Typ-2-Diabetikern mit Alzheimer-Risiko sind. Diese Ergebnisse eröffnen die Möglichkeit für neue Prozeduren in der klinischen Versorgung, die auf die Überprüfung des kognitiven Status ausgerichtet sind und die sich leicht in die augenärztlichen Routineuntersuchungen zum Screening auf DR integrieren lassen.

\section{Einfluss der DR auf das Management von Diabetes- Patienten}

Zwar besteht kein Zweifel, dass es zwischen der Blutzuckereinstellung und der langfristigen Entwicklung und Progression einer DR einen Zusammenhang gibt, doch kommt es Berichten zufolge bei rascher Verbesserung einer Hyperglykämie zu einer initialen Verschlechterung der DR. In der DCCT (Diabetes Control and Complications Trial)-Studie wurde bei 13,1\% von 711 Typ-1-Diabetikern unter intensivierter Therapie eine frühe Verschlechterung nach 6 und/oder 12 Monaten beobachtet, gegenüber 7,6\% von 728 Patienten, die die konventionelle Therapie erhielten (Odds Ratio: 2,06; $p<0,001$ ) [53]. Die Hauptrisikofaktoren für eine frühe Verschlechterung waren ein hoher HbAlc-Wert, eine starke (> 2\%) und rasche Senkung des HbAlc-Werts und der Schweregrad der DR bei Studienbeginn [53-55]. Ein ähnliches Phänomen wurde für Typ-2-Diabetiker nach einer raschen Verbesserung der Blutzuckerwerte berichtet, wenn die Patienten von oralen Wirkstoffen oder einer alleinigen Ernährungstherapie auf eine Behandlung mit Insulin umgestellt wurden [56, 57], sowie laut veröffentlichten Berichten nach einer bariatrischen Operation [58].

Interessanterweise manifestiert sich die initiale Verschlechterung der DR, wie sie in der Frühphase einer intensivierten Therapie zur Blutzuckereinstellung zu beobachten ist, durch vermehrte weiche Exsudate, einem typischen Merkmal der ischämischen Retina. Die Hypoglykämie verschlimmert die ischämische Netzhautschädigung [59] und Antidiabetika, bei denen, wenn überhaupt, nur ein sehr geringes Hypoglykämierisiko besteht, wie beispielsweise GLP-1RA oder SGLT-2-Inhibitoren, könnten theoretisch für die Einleitung einer intensivierten Therapie bei Patienten mit DR besser geeignet sein.

Der enge Zusammenhang zwischen der Senkung der HbAlcWerte und den günstigen Effekten auf die DR hat überdeckt, dass klinische Studien erforderlich sind, um den spezifischen Effekt von Antidiabetika auf die DR per se unabhängig von ihrer blutzuckersenkenden Wirksamkeit zu untersuchen. Aufgrund der berichteten pleiotropen Aktivität von GLP-1RA in experimentellen DR-Modellen verfügen diese Arzneimittel - neben ihrer Fähigkeit, den Blutzucker zu senken - über einen potenziellen zusätzlichen Nutzen, indem sie die Entwicklung einer DR verhindern oder das Fortschreiten einer DR stoppen [60]. Die Ergebnisse der SUSTAIN-6-Studie bilden diesbezüglich eine Ausnahme. In dieser Studie zeigte Semaglutid (ein langwirksamer GLP-1-Rezeptoragonist) unerwartet eine höhere Rate schwerer DR-Komplikationen (d.h. Glaskörperblutungen, Erblindung oder Erkrankungen, die eine Behandlung mit einem intravitrealen Wirkstoff oder eine Photokoagulation erforderten) [61]. Obwohl dies bei einem sehr geringen Prozentsatz von Patienten beobachtet wurde, handelte es sich dabei um ein überraschendes Ergebnis, da in den präklinischen Studien zu GLP-1RA - wie oben erwähnt - eher günstige Effekte als schädliche Effekte auf die Entwicklung einer DR berichtet wurden [60]. Hauptgründe für die schädliche Wirkung waren der rasche Abfall der HbAlcWerte und die fehlende Einstufung der DR bei Studieneintritt [60, 62]. Eine spezielle klinische Studie (die FOCUS-Studie), die darauf ausgerichtet ist, Klarheit in Bezug auf dieses Problem zu schaffen, wird jedoch in Kürze gestartet.

\section{Abschließende Bemerkungen und zukünftige Perspektiven}

Trotz der Verbesserungen beim Diabetes-Management ist die DR in den Industrieländern weiterhin eine der Hauptursachen für eine Minderung der Sehkraft und Erblindung der Bevölkerung im arbeitsfähigen Alter. Erblindung und Verlust der Sehkraft, die größten Ängste der meisten Diabetiker, führen zu emotionaler Belastung und verringerter Funktionsfähigkeit im Alltag [63]. Darüber hinaus kommt es Berichten zufolge in sehr frühen Stadien der DR, in denen lediglich eine Neurodegeneration vorliegt, zu Veränderungen der Lebensqualität [64]. Daher sollten die jahrzehntealten Systeme zur Einstufung der DR, die allein auf einer nicht quantitativen Einschätzung der mikrovaskulären Anoma- 
lien basieren, durch robuste quantitative Messungen ersetzt werden, welche die fortschreitende Funktionsstörung der retinalen neurovaskulären Einheit widerspiegeln. Ferner sind stärker interventionell ausgerichtete Studien erforderlich, in denen die pathogenetischen Mechanismen untersucht werden, welche die retinale neurovaskuläre Einheit beeinflussen und sowohl eine Vaso- als auch Neuroprotektion bewirken. Dies wird nicht nur für ein besseres Verständnis der DR von entscheidender Bedeutung sein, sondern auch für die Umsetzung eines Ansatzes zur frühzeitigen und effizienten personalisierten Therapie in der Behandlung dieser Erkrankung.

Einem wichtigen Konzept zufolge ist die DR nicht nur mit anderen diabetischen mikroangiopathischen Erkrankungen wie Nephropathie oder Neuropathie assoziiert, sondern auch mit kardiovaskulären Erkrankungen. Dieses etablierte Konzept sollte nicht nur in der klinischen Praxis berücksichtigt werden, sondern auch bei der Konzeption von Studien, die auf eine Beurteilung des kardiovaskulären Outcomes in der Diabetiker-Population ausgerichtet sind.

Die DR ist zudem mit Demenz assoziiert [49]; noch interessanter ist jedoch der seit kurzem vorliegende Nachweis, dass die Untersuchung der retinalen neuronalen Funktionsstörung helfen kann, Typ-2-Diabetiker zu identifizieren, die sich in einem Prodromalstadium der Demenz (d.h. MCI) befinden. Dies ist klinisch relevant, da bei einer frühzeitigen Erkennung einer kognitiven Ein- schränkung die Therapieziele und die Behandlung der Patienten angepasst werden sollten, um Hypoglykämien, Hospitalisierungen und eine Akzeleration der Demenz zu vermeiden. Ferner ist zu beachten, dass Interventionen, welche die klinische Manifestation der Demenz um ein Jahr verzögern, die Prävalenz um 9 Millionen Fälle im Jahr 2050 verringern würden [65].

Insgesamt bedeutet das Vorliegen einer DR bei einem Diabetiker nicht nur, dass ein «Problem mit dem Sehen» besteht, sondern auch, dass dieser Patient ein hohes Risiko hat, andere mikro- und makrovaskläre Erkrankungen sowie eine Demenz zu entwickeln. Insofern ist die DR ein häufig vernachlässigter kennzeichnender Faktor bei der Umsetzung einer personalisierten Therapie bei Diabetikern. Darüber hinaus könnte die Retina ein hilfreiches Fenster für die indirekte Untersuchung des Gehirns darstellen, wodurch es möglich wäre, Patienten mit kognitiven Einschränkungen und dadurch bedingtem Demenzrisiko zu identifizieren. Koordinierte Aktivitäten von Diabetologen, Ophthalmologen, Grundlagenforschern, pharmazeutischen Unternehmen und Ärzten sind entscheidend für eine Linderung der verheerenden Auswirkungen dieser immer noch hochprävalenten Komplikation von Diabetes mellitus.

\section{Disclosure Statement}

Die Autoren haben keine Interessenkonflikte offenzulegen.

\section{References}

1 Antonetti DA, Klein R, Gardner TW: Diabetic retinopathy. N Engl J Med 2012;366:1227-1239.

-2 Yau JW, Rogers SL, Kawasaki R, et al.: MetaAnalysis for Eye Disease (META-EYE) Study Group. Global prevalence and major risk factors of diabetic retinopathy. Diabetes Care 2012;35:556-564.

>3 Wong TY, Cheung CM, Larsen M, et al.: Diabetic retinopathy. Nat Rev Dis Primers 2016;2: 16012.

4 Simó R, Hernández C, European Consortium for the Early Treatment of Diabetic Retinopathy (EUROCONDOR): Neurodegeneration in the diabetic eye: new insights and therapeutic perspectives. Trends Endocrinol Metab 2014; 25:23-33.

5 Simó R, Stitt AW, Gardner TW: Neurodegeneration in diabetic retinopathy: does it really matter? Diabetologia 2018;61:1902-1912.

6 Solomon SD, Chew E, Duh EJ, et al.: Diabetic retinopathy: a position statement by the American Diabetes Association. Diabetes Care 2017; 40:412-418.

7 Heintz E, Wiréhn AB, Peebo BB, et al.: Prevalence and healthcare costs of diabetic retinopathy: a population-based register study in Sweden. Diabetologia 2010;53:2147-254.

8 International Diabetes Federation: IDF Diabetes Atlas 2017, www.diabetesatlas.org.

9 Simó R, Hernández C: Novel approaches for treating diabetic retinopathy based on recent pathogenic evidence. Prog Retin Eye Res 2015; 48:160-180.
10 Stitt AW, Curtis TM, Chen M, et al.: The progress in understanding and treatment of diabetic retinopathy. Prog Retin Eye Res 2016;51: $156-186$.

11 UK Prospective Diabetes Study Group: Tight blood pressure control and risk of macrovascular and microvascular complications in type 2 diabetes: UKPDS 38. BMJ 1998;317: 703-713.

12 Nathan DM, Genuth S, Lachin J, et al.: The effect of intensive treatment of diabetes on the development and progression of long-term complications in insulin-dependent diabetes mellitus. N Engl J Med 1993;329:977-986.

13 Nordwall M, Abrahamsson M, Dhir M, et al.: Impact of $\mathrm{HbAlc}$, followed from onset of type 1 diabetes, on the development of severe retinopathy and nephropathy: the VISS Study (Vascular Diabetic Complications in Southeast Sweden). Diabetes Care 2015;38:308-315.

14 Sacks FM, Hermans MP, Fioretto P, et al.: Association between plasma triglycerides and high-density lipoprotein cholesterol and microvascular kidney disease and retinopathy in type 2 diabetes mellitus: a global case-control study in 13 countries. Circulation. 2014;129: 999-1008.

15 Klein BE, Myers CE, Howard KP, et al.: Serum lipids and proliferative diabetic retinopathy and macular edema in persons with long-term type 1 diabetes mellitus: The Wisconsin Epidemiologic Study of Diabetic Retinopathy. JAMA Ophthalmol 2015;133:503-510.
16 Sasongko MB, Wong TY, Nguyen TT, et al. Serum apolipoprotein AI and B are stronger biomarkers of diabetic retinopathy than traditional lipids. Diabetes Care 2011;34:474-479.

17 Sasongko MB, Wong TY, Nguyen TT, et al.: Novel versus traditional risk markers for diabetic retinopathy. Diabetologia 2012;55: 666-670.

18 Liu Y, Yang J, Tao L, et al.: Risk factors of diabetic retinopathy and sight-threatening diabetic retinopathy: a cross-sectional study of 13 473 patients with type 2 diabetes mellitus in mainland China. BMJ Open 2017;7:e016280.

19 Armstrong C, Joint National Committee: JNC8 guidelines for the management of hypertension in adults. Am Fam Physician 2014;90: 503-504.

20 Vujosevic S, Simó R: Local and systemic inflammatory biomarkers of diabetic retinopathy: an integrative approach. Invest Ophthalmol Vis Sci 2017;58:BIO68-75.

21 Lachin JM, Genuth S, Nathan DM, et al.: Effect of glycemic exposure on the risk of microvascular complications in the diabetes control and complications trial-revisited. Diabetes 2008; 57:995-1001.

-22 Arar NH, Freedman BI, Adler SG, et al.: Heritability of the severity of diabetic retinopathy: the FIND-Eye study. Invest Ophthalmol Vis Sci 2008;49:3839-3845.

23 Hietala K, Forsblom C, Summanen P, et al.: Heritability of proliferative diabetic retinopathy. Diabetes 2008;57:2176-2180. 
24 Leslie RD, Pyke DA: Diabetic retinopathy in identical twins. Diabetes. 1982;31:19-21.

-25 The Diabetes Control and Complications Trial Research Group: Clustering of long-term complications in families with diabetes in the diabetes control and complications trial. Diabetes 1997;46:1829-1839.

-26 Rema M, Saravanan G, Deepa R, et al.: Familial clustering of diabetic retinopathy in South Indian Type 2 diabetic patients. Diabet Med 2002;19:910-916.

-27 Hallman DM, Huber JC Jr, Gonzalez VH, et al.: Familial aggregation of severity of diabetic retinopathy in Mexican Americans from Starr County, Texas. Diabetes Care 2005;28: 1163-1168.

28 Monti MC, Lonsdale JT, Montomoli C, et al.: Familial risk factors for microvascular complications and differential male-female risk in a large cohort of American families with type 1 diabetes. J Clin Endocrinol Metab 2007;92: 4650-4655.

$29 \mathrm{Lu}$ J, Ma X, Zhou J, et al.: Association of time in range, as assessed by continuous glucose monitoring, with diabetic retinopathy in type 2 diabetes. Diabetes Care 2018;41:2370-2376.

-30 Zhao Q, Zhou F, Zhang Y, et al.: Fasting plasma glucose variability levels and risk of adverse outcomes among patients with type 2 diabetes: a systematic review and meta-analysis. Diabetes Res Clin Pract 2019;148:23-31.

-31 Reddy MA, Zhang E, Natarajan R: Epigenetic mechanisms in diabetic complications and metabolic memory. Diabetologia 2015;58: 443-455.

\$2 Pearce I, Simó R, Lövestam-Adrian M, et al. Association between diabetic eye disease and other complications of diabetes: implications for care. A systematic review. Diabetes Obes Metab 2019;21:467-478.

33 Rossing P, Hougaard P, Parving HH: Risk factors for development of incipient and overt diabetic nephropathy in type 1 diabetic patients: a 10-year prospective observational study. Diabetes Care 2002;25:859-864.

34 Parving HH, Lewis JB, Ravid M, et al.: Prevalence and risk factors for microalbuminuria in a referred cohort of type II diabetic patients: a global perspective. Kidney Int 2006;69: 2057-2063.

35 Karlberg C, Falk C, Green A, et al.: Proliferative retinopathy predicts nephropathy: a 25 year follow-up study of type 1 diabetic patients. Acta Diabetol 2012;49:263-268.

-36 Moriya T, Tanaka S, Kawasaki R, et al.: Diabetic retinopathy and microalbuminuria can predict macroalbuminuria and renal function decline in Japanese type 2 diabetic patients: Japan Diabetes Complications Study. Diabetes Care 2013;36:2803-2809.
7 Rossing K, Christensen PK, Hovind Pet al.: Progression of nephropathy in type 2 diabetic patients. Kidney Int 2004;66:1596-1605.

38 Kostev K, Jockwig A, Hallwachs A, et al.: Prevalence and risk factors of neuropathy in newly diagnosed type 2 diabetes in primary care practices: a retrospective database analysis in Germany and U.K. Prim Care Diabetes 2014;8: 250-255.

39 Lin IC, Wang YH, Lin CL, et al.: Diabetic polyneuropathy and the risk of developing diabetic retinopathy: a nationwide, population-based study. Acta Ophthalmol 2015;93:713-718.

40 Huang CC, Lee JJ, Lin TK, et al.: Diabetic retinopathy is strongly predictive of cardiovascular autonomic neuropathy in type 2 diabetes. J Diabetes Res 2016;2016:6090749.

41 Voulgari C, Psallas M, Kokkinos A, et al.: The association between cardiac autonomic neuropathy with metabolic and other factors in subjects with type 1 and type 2 diabetes. J Diabetes Complications 2011;25:159-167.

42 Pemp B, Palkovits S, Howorka K, et al.: Correlation of retinal neurodegeneration with measures of peripheral autonomic neuropathy in type 1 diabetes. Acta Ophthalmol 2018; 96:e804-10.

43 Brownrigg JR, Hughes CO, Burleigh D, et al.: Microvascular disease and risk of cardiovascular events among individuals with type 2 diabetes: a population-level cohort study. Lancet Diabetes Endocrinol 2016;4:588-597.

44 U.S. Food and Drug Administration. Guidance for industry: Diabetes mellitus - evaluating cardiovascular risk in new antidiabetic therapies to treat type 2 diabetes. www.fda.gov/ downloads/Drugs/GuidanceComplianceRegulatoryInformation/Guidance/ucm071627.pdf.

45 Davies MJ, D'Alessio DA, Fradkin J, et al.: Management of hyperglycemia in type 2 diabetes, 2018. a consensus report by the American Diabetes Association (ADA) and the European Association for the Study of Diabetes (EASD). Diabetes Care 2018;41:2669-2701.

46 Koekkoek PS, Kappelle LJ, van den Berg E, et al: Cognitive function in patients with diabetes mellitus: guidance for daily care. Lancet Neurol 2015; 14:329-340.

47 Biessels GJ, Staekenborg S, Brunner E, et al.: Risk of dementia in diabetes mellitus: a systematic review. Lancet Neurol 2006;5:64-74.

48 Ciudin A, Espinosa A, Simó-Servat O, et al.: Type 2 diabetes is an independent risk factor for dementia conversion in patients with mild cognitive impairment. J Diabetes Complications 2017;31:1272-1274.

49 Simó R, Ciudin A, Simó-Servat O, et al.: Cognitive impairment and dementia: a new emerging complication of type 2 diabetes-The diabetologist's perspective. Acta Diabetol 2017;54: 417-424.
American Diabetes Association: 6. Glycemic Targets. Diabetes Care 2017;40 Suppl 1:S48-56.

51 Ciudin A, Simó-Servat O, Hernández C, et al.: Retinal microperimetry: A new tool for identifying patients with type 2 diabetes at risk for developing Alzheimer disease. Diabetes 2017; 66:3098-3104.

52 Simó-Servat O, Ciudin A, Ortiz-Zúñiga ÁM, et al.: Usefulness of eye fixation assessment for identifying type 2 diabetic subjects at risk of dementia. J Clin Med 2019;8:E59.

53 Early worsening of diabetic retinopathy in the Diabetes Control and Complications Trial. Arch Ophthalmol 1998;116:874-886.

54 Funatsu H, Yamashita H, Ohashi Y, et al.: Effect of rapid glycemic control on progression of diabetic retinopathy. Jpn J Ophthalmol 1992;36:356-367.

55 Shurter A, Genter P, Ouyang D, et al.: Euglycemic progression: worsening of diabetic retinopathy in poorly controlled type 2 diabetes in minorities. Diabetes Res Clin Pract 2013;100 362-367.

56 Henricsson M, Nilsson A, Janzon L, et al: The effect of glycaemic control and the introduction of insulin therapy on retinopathy in noninsulin-dependent diabetes mellitus. Diabet Med 1997;14:123-131.

57 Roysarkar TK, Gupta A, Dash RJ, et al.: Effect of insulin therapy on progression of retinopathy in noninsulin-dependent diabetes mellitus. Am J Ophthalmol 1993;115:569-574.

58 Gorman DM, le Roux CW, Docherty NG: The effect of bariatric surgery on diabetic retinopathy: Good, bad, or both? Diabetes Metab J 2016;40:354-364

59 Casson RJ, Wood JP, Osborne NN: Hypoglycaemia exacerbates ischaemic retinal injury in rats. Br J Ophthalmol 2004;88:816-820.

60 Simó R, Hernández C: GLP-1R as a target for the treatment of diabetic retinopathy: friend or foe? Diabetes 2017;66:1453-1460.

61 Marso SP, Bain SC, Consoli A, et al.: SUSTAIN-6 Investigators. Semaglutide and cardiovascular outcomes in patients with type 2 diabetes. N Engl J Med 2016;375:1834-1844.

62 Vilsbøll T, Bain SC, Leiter LA, et al.: Semaglutide, reduction in glycated haemoglobin and the risk of diabetic retinopathy. Diabetes Obes Metab 2018;20:889-897.

63 Fong DS, Aiello LP, Ferris FL 3rd, et al.: Diabetic retinopathy. Diabetes Care 2004;27: 2540-2553.

64 Trento M, Durando O, Lavecchia S, et al.: Vision related quality of life in patients with type 2 diabetes in the EUROCONDOR trial. Endocrine 2017;57:83-88.

65 Hampel H, Frank R, Broich K, et al.: Biomarkers for Alzheimer's disease: academic, industry and regulatory perspectives. Nat Rev Drug Discov 2010;9:560-574. 